\title{
Nogo-B receptor increases the resistance to tamoxifen in estrogen receptor-positive breast cancer cells
}

\author{
Pin Gao ${ }^{1,2,3}$, Xiang Wang ${ }^{2,3,4}$, Ying Jin ${ }^{1,2,3}$, Wenquan $\mathrm{Hu}^{2,3}$, Yajun Duan ${ }^{2,3,4}$, Aiping Shi ${ }^{1}$, Ye Du', Dong Song ${ }^{1}$, \\ Ming Yang ${ }^{1}$, Sijie Li ${ }^{1}$, Bing Han ${ }^{1}$, Gang Zhao ${ }^{1}$, Hongquan Zhang ${ }^{4,5^{*}}$, Zhimin Fan ${ }^{1,5^{*}}$ and Qing Robert Miao ${ }^{2,3,5^{*}}$ (D)
}

\begin{abstract}
Backgrounds: Tamoxifen is typically used to treat patients with estrogen receptor alpha (ERa)-positive breast cancer. However, 30\% of these patients gain acquired resistance to tamoxifen during or after tamoxifen treatment. As a Ras modulator, Nogo-B receptor (NgBR) is required for tumorigenesis through the signaling crosstalk with epidermal growth factor (EGF) receptor (EGFR)-mediated pathways. NgBR is highly expressed in many types of cancer cells and regulates the sensitivity of hepatocellular carcinoma to chemotherapy. In this study, we found the expression of $\mathrm{NgBR}$ is increased in tamoxifen-resistant ERa-positive breast cancer cells.
\end{abstract}

Methods: Tamoxifen-resistant ERa-positive MCF-7 and T47D breast cancer cell lines were established by culturing with gradually increased concentration of 4-hydroxytamoxifen (4-OHT). The effects of NgBR on tamoxifen resistance was determined by depleting $\mathrm{NgBR}$ in these cell lines using previously validated small interfering RNA (siRNA). The effects of 4-OHT on cell viability and apoptosis were determined using well-accepted methods such as clonogenic survival assay and Annexin V/propidium iodide staining. The alteration of EGF-stimulated signaling and gene expression was determined by western blot analysis and real-time PCR, respectively.

Results: NgBR knockdown with siRNA attenuates EGF-induced phosphorylation of ERa and restores the sensitivity to tamoxifen in ERa-positive breast cancer cells. Mechanistically, our data demonstrated that NgBR knockdown increases the protein levels of p53 and decreases survivin, which is an apoptosis inhibitor.

Conclusions: These results suggested that $\mathrm{NgBR}$ is a potential therapeutic target for increasing the sensitivity of ERapositive breast cancer to tamoxifen.

Keywords: Nogo-B receptor, Survivin, Tamoxifen, Estrogen receptor, Breast cancer

\section{Background}

Breast cancer is the most common cancer in women around the world $[1,2]$. About $75 \%$ of the cases are estrogen receptor alpha (ER $\alpha)$-positive breast cancer [3]. These patients undergo adjuvant endocrine therapy to increase

\footnotetext{
*Correspondence: hongquan.zhang@bjmu.edu.cn; fanzhimn@163.com; qmiao@mcw.edu

${ }^{4}$ Department of Human Anatomy, Histology, and Embryology, Key Laboratory of Carcinogenesis and Translational Research (Ministry of Education) and State Key Laboratory of Natural and Biomimetic Drugs, Peking University Health Science Center, Beijing 100191, China 'Department of Breast Surgery, The First Hospital of Jilin University, 71 Xinmin street, Changchun 130021, Jilin Province, China

${ }^{2}$ Division of Pediatric Surgery, Department of Surgery, Children's Research Institute, Medical College of Wisconsin, 8701 W Watertown Plank Rd, Milwaukee, WI 53226, USA

Full list of author information is available at the end of the article
}

disease-free survival (DFS) and overall survival (OS) [4]. According to the National Comprehensive Cancer Network (NCCN) guideline, patients with invasive breast cancer who are ER $\alpha$-positive or progesterone receptor (PR)-positive are eligible for tamoxifen, the selective estrogen receptor modulator (SERM) [5]. Tamoxifen, or its active metabolite 4-hydroxytamoxifen (4-OHT), are reported to induce breast cancer cell apoptosis [6]. However, recurrence within 15 years occurs in one third of patients treated with tamoxifen for 5 years [4].

The underlying mechanisms of developing resistance, especially acquired resistance, to tamoxifen are complex and numerous, including ligand-independent activation

(c) The Author(s). 2018 Open Access This article is distributed under the terms of the Creative Commons Attribution 4.0 International License (http://creativecommons.org/licenses/by/4.0/), which permits unrestricted use, distribution, and reproduction in any medium, provided you give appropriate credit to the original author(s) and the source, provide a link to the Creative Commons license, and indicate if changes were made. The Creative Commons Public Domain Dedication waiver (http://creativecommons.org/publicdomain/zero/1.0/) applies to the data made available in this article, unless otherwise stated. 
of ER $\alpha$ or its co-activators through phosphorylation, and the inhibition of apoptosis through constitutive activation of survival signaling [7]. Clinical evidence shows that patients with human epidermal growth factor receptor 2 (HER2) overexpression and lower ER $\alpha$ levels are more likely to become tamoxifen-resistant [8]. Preclinical studies implicate the contribution of growth factor receptor signaling pathways, such as EGFR and HER2, to tamoxifen resistance $[9,10]$.

Nogo isoforms, including Nogo-A, Nogo-B and Nogo- $C$, are members of a reticulon protein superfamily. Nogo-B is mainly expressed in peripheral tissues [11]. $\mathrm{NgBR}$ was identified as a Nogo-B receptor specific for the amino terminus of Nogo-B (AmNogo-B). NgBR is necessary for angiogenesis by mediating chemotaxis of endothelial cells [12], and is essential for vasculature development $[13,14]$. Our recent findings demonstrated that NgBR binds farnesylated Ras and recruits Ras to the plasma membrane, which is a critical step required for receptor tyrosine kinase (RTK)-mediated activation of Ras signaling in human breast cancer cells and tumorigenesis [15]. Greater expression of NgBR in ER $\alpha$-positive breast tumor tissues is significantly correlated with expression of survivin [16], which is an apoptosis inhibitor [17]. A proteomic study also showed that NgBR is essential for promoting epithelial-mesenchymal transition (EMT) in breast cancer cells [18]. However, the involvement of $\mathrm{NgBR}$ in tamoxifen resistance of ER $\alpha$-positive breast cancer is still unknown. In this study, we showed that NgBR knockdown attenuates tamoxifen resistance in MCF-7 and T47D breast cancer cells by inhibiting EGF-stimulated phosphorylation of ER $\alpha$. Also, NgBR knockdown restored the sensitivity of ER $\alpha$-positive breast cancer cells to tamoxifen through decreasing p53-mediated expression of survivin. Our results suggest that $\mathrm{NgBR}$ is a potential therapeutic target for increasing the efficacy of tamoxifen and overcoming the resistance to tamoxifen in ER $\alpha$-positive patients with breast cancer.

\section{Methods}

Antibodies, reagents and plasmids

A peptide (AHHRMRWRADGRSLEK, residues from 81 to 96 of $\mathrm{NgBR}$ ) was used to immunize rabbits (Epitomics, Burlingame, CA, USA). Antiserum was purified using the same peptide-conjugated SulfoLink Coupling Gel (Pierce, Rockford, IL, USA). Purified NgBR rabbit polyclonal antibody was used for immunostaining. The peptide recognizing epitope 14-30 of human Nogo-B was used to immunize rabbits (IMG-5346A, Imgenex, San Diego, CA, USA). Antibodies for NgBR (\#ab168351) and phosphorylated ER $\alpha$ (phos-S118) (\#ab32396) were purchased from Abcam (Cambridge, MA, USA). Antibodies for phos-EGFR (\#3777), phos-Akt (\#S473), phos-p42/44 extracellular signal-related kinase (ERK)
(\#9101), total Akt (\#4691), total ERK (\#4595), ER $\alpha$ (\#8644), HER2 (\#4290) and survivin (\#2808) were purchased from Cell Signaling Technology (Beverly, MA, USA). We also used antibodies for p53 (\#10442-1-AP), $\beta$-actin (\#60008-1) and Hsp90 (13171-1-AP) from Proteintech (Rosement, IL, USA). EGF (\#E5036) was purchased from Sigma-Aldrich (St. Louis, MO, USA). HER2 plasmid DNA was a gift from Mien-Chie Hung (Addgene plasmid \# 16257).

\section{Cell culture}

Human breast cancer cell lines MCF-7 and T-47D were obtained from the American Type Culture Collection. The cells were grown in DMEM with L-Glutamine (MCF-7 Gibco) or RPMI-1640 with L-Glutamine (T47D, Gibco) supplemented with 10\% FBS (Sigma, St. Louis, MO, USA) and $1 \%$ penicillin streptomycin glutamine (Gibco). The tamoxifen-resistant cell lines (MCF-7TamR and T47D-TamR) were derived from the parental cell lines by continuous gradual exposure to 4-OHT (Sigma, St. Louis, MO, USA) to reach the final concentration of $1 \mu \mathrm{M}$ in methanol in 6 months [19]. Culture medium was replaced every 2-3 days. Cells were incubated at $37{ }^{\circ} \mathrm{C}$ in a humidified atmosphere containing $5 \% \mathrm{CO} 2$. The cell lots used in this study were authenticated prior to in vitro experiments.

\section{Small interfering RNA (siRNA) and plasmid transfection} NgBR siRNA (forward, GGAAAUACAUAGACCUACA; reverse, UGUAGGUCUAUGUAUUUCC) oligonucleotides with 3' dTdT overhangs were synthesized by QIAGEN (Valencia, CA, USA). The specificity of NgBR siRNA has been validated in our previous publication [12]. Control siRNA in experiments refers to a non-silencing (NS) siRNA (NSF, UUCUCCGAACGUGUCACGU; NSR, AC GUGACACGUUCGG AGAA) designed and synthesized by QIAGEN. P53 siRNA (sc44218) and survivin siRNA (sc-29,499) were purchased from Santa Cruz (Dallas, TX, USA). Cells were transfected with siRNA using Lipofectamine RNAiMAX reagent (ThermoFisher Scientific). For transient NgBR overexpression experiments, MCF-7 cells were transfected with pIRES-NgBR-HA plasmid DNA using Lipofectamine 2000 reagent (ThermoFisher Scientific).

\section{Quantitative real-time polymerase chain reaction}

Total RNA was extracted from cells by using TRIzol reagent according to the manual (ThermoFisher Scientific) and complementary DNA (cDNA) was reverse-transcribed using the iScript cDNA Synthesis Kit (Bio-Rad, Hercules, CA, USA) according to the manufacturer's instructions. Real-time PCR was performed using iTaq Universal SYBR Green Supermix (Bio-Rad, USA) and was run on MyiQ Single Color Real-Time PCR Detection System (Bio-Rad). The relative messenger RNA (mRNA) expression of each 
gene was normalized to glyceraldehyde-3-phosphate dehydrogenase (GAPDH) RNA levels. The primers were synthesized by Integrated DNA Technologies (Coralville, IA, USA). The forward and reverse primers for $\mathrm{NgBR}$ are $5^{\prime}$-tgccagttagtagcccagaagcaa-3' and 5'-tgatgtgccagggaagaaagccta- $3^{\prime}$, respectively. The forward and reverse primers for p53 are $5^{\prime}$-cctttctatcagccccagaggata-3' and 5'-GGGA CATCCTTAATTATCTGGGGT-3', respectively. The forward and reverse primers for GAPDH are $5^{\prime}$-aacctgccaagtatgatgac-3' and $5^{\prime}$-tctcttgctcagtgtccttg-3', respectively. The forward and reverse primers for EGFR are $5^{\prime}$-aagccatatgacggaatccc- $3^{\prime}$ and $5^{\prime}$-ggaactttgggcgactatctg-3', respectively. The forward and reverse primers for ER $\alpha$ are $5^{\prime}$-cgactatatgtgtccagccac-3' and 5' -cctcttcggtcttttcgtatcc-3', respectively. The forward and reverse primers for survivin are $5^{\prime}$-caaggagctggaaggctg- $3^{\prime}$ and $5^{\prime}$-ttcttggctctttctctgtcc- $3^{\prime}$, respectively.

\section{Clonogenic survival assay}

Cells were seeded in triplicate into cell culture dishes (1000 cells/well). MCF-7-TamR or T47D-TamR cells were transfected with NgBR siRNA or non-silencing siRNA. At $12 \mathrm{~h}$ after transfection, cells were treated with or without 4-OHT. After 14 days, cells were washed with PBS, fixed with methanol for $15 \mathrm{~min}$ and stained with $0.1 \%$ crystal violet for $15 \mathrm{~min}$. Colonies containing 50 or more cells were counted [20].

\section{Cell viability assay}

Cell viability was determined using the CCK-8 assay. Cells were seeded at the density of 5000 cells/well into 96-well plates; $10 \mu \mathrm{L}$ of CCK-8 (Sigma) was added to each well. Then cells were incubated at $37{ }^{\circ} \mathrm{C}$ for $1-3 \mathrm{~h}$. The absorbance of the reaction was measured using a plate reader (Molecular Devices, Sunnyvale, CA, USA). Another cell viability assay used in this study was trypan blue staining. Cells were seeded into 96-well plates (5000/well). Then, the cells were incubated for $24 \mathrm{~h}$ before transfection with NgBR siRNA. The treatment time with 4-OHT was 3 days. Before staining, cells were washed with PBS and dislodged with trypsin. Then cell suspension and trypan blue were mixed (1:1) for $3 \mathrm{~min}$. The number of viable and dead cells on the hemocytometer were counted under a microscope.

\section{Apoptosis assay by FITC Annexin V/propidium iodide (PI) staining}

An FITC Annexin V Apoptosis Detection kit (\# 556547) was purchased from BD Biosciences (San Jose, CA, USA). Cells cultured in 6-well plates were transfected with siRNA of NgBR, p53 or survivin, and then treated with 4-OHT for $48 \mathrm{~h}$. Cells were then stained with $5 \mu \mathrm{L}$ Annexin V-FITC and $5 \mu \mathrm{L}$ PI in $500 \mathrm{uL}$ of apoptosis reaction solution at room temperature in the dark for
15 min following the manufacture's instruction. The BD LSR II flow cytometer was used to detect apoptotic cells.

\section{Western blot}

Cells were harvested and washed with PBS, and then lysed with lysis buffer supplemented with Pierce Protease and Phosphatase Inhibitor Mini Tablets (ThermoFisher Scientific) for $10 \mathrm{~min}$ on ice. The whole cell lysate was scraped from the plates and then centrifuged at $12000 \mathrm{rpm}$ for 10 min at $4{ }^{\circ} \mathrm{C}$. The concentrations of protein were determined by BCA Protein Assay Kit (Bio-Rad). Cell lysates were subjected to SDS-PAGE, transferred to nitrocellulose blotting membrane (GE Healthcare Life Sciences, Pittsburgh, PA, USA), then incubated with primary specific antibodies at $4{ }^{\circ} \mathrm{C}$ overnight. The membranes were incubated with secondary antibodies (Jackson ImmunoResearch, West Grove, PA, USA) at 1:10000 dilution for $2 \mathrm{~h}$ at room temperature. The protein band intensities were evaluated using Amersham ECL Western Blotting Detection System (GE Healthcare Life Sciences) and were normalized to housekeeping genes, either $\beta$-actin or HSP90. All western blot experiments were performed at least three times.

\section{Raf-pull-down assay}

Ras activity was assessed using GST-Raf-1 RBD beads (RF02, Cytoskeleton, Denver, CO, USA) according to the manufacturer's protocol: $500 \mu \mathrm{g}$ total cell lysate was incubated with $10 \mu \mathrm{L}$ GST-Raf1-RBD beads overnight at $4{ }^{\circ} \mathrm{C}$ with gentle rocking. Samples were washed five times, then dissolved in $20 \mu \mathrm{L} 2 \mathrm{X}$ SDS sample buffer. Activated $\mathrm{H}$-Ras and K-Ras was determined by western blot using specific H-Ras (GTX116041, GeneTex, San Antonio, TX, USA) or K-Ras (12063-1-AP, Proteintech, Rosemont, IL, USA) antibodies, respectively.

\section{Tissue microarray slides}

Breast cancer tissue was collected from 22 patients at the First Hospital of Jilin University (Changchun, China).

We had consent from all patients for participating in this study. All of the patients received modified radical mastectomy and were diagnosed with infiltrating ductal carcinoma via pathological diagnosis. Immunohistochemistry was performed to examine NgBR, Nogo-B, and survivin expression levels following standard methodology described in our previous publication [16]. All of these breast cancer cases were histopathologically re-evaluated on hematoxylin and eosin-stained slides by two pathologists. The breast tissue specimens are anonymous. The study was approved by the ethical committee of the First Hospital of Jilin University. 


\section{Association between $\mathrm{NgBR}$ or survivin expression and survival in patients with breast cancer}

All data were collected from a public online clinical database (http://kmplot.com). We analyzed the association between mRNA level of NgBR (NUS1, 225071_x at from Kaplan-Meier Plot database) or survivin (BIRC5, 202094_x at from Kaplan-Meier Plot database) and survival in patients with breast cancer. Kaplan-Meier survival curves according to NgBR expression status were used to analyze the relapse-free survival (RFS) and $\log$-rank $p$ values (SPSS 23.0 USA).

\section{Statistical analysis}

Data were analyzed from at least three independent experiments. The results were reported as the mean \pm SD . Values of $p<0.05$ were considered statistically significant. Student's $t$ test or analysis of variance (ANOVA) were performed as appropriate. Correlation between $\mathrm{NgBR}$ and survivin expression was analyzed using Fisher's test. Statistical analyses were performed using Prism 6.0 software (GraphPad software, USA).

\section{Results}

$\mathrm{NgBR}$ expression is increased in tamoxifen-resistant breast cancer cells

Tamoxifen resistant MCF-7 (MCF-7-TamR) and T47D (T47D-TamR) ER $\alpha$-positive breast cancer cells were established following the previously described method [19]. To validate tamoxifen resistance in established MCF-7-TamR and T47D-TamR cells, both normal and tamoxifen-resistant cells were treated with $0-5 \mu \mathrm{M} 4-\mathrm{OHT}$. As shown in Fig. 1a-d, $5 \mu \mathrm{M}$ 4-OHT cannot attenuate the colony formation capability of MCF-7-TamR and T47D-TamR cells. However, parental cells cannot survive treatment with $5 \mu \mathrm{M}$ 4-OHT. CCK-8 cell viability assay was also used for determining the response of these breast cancer cells to tamoxifen (Additional file 1: Figure S1A and B). Similarly, both MCF-7-TamR and T47D-TamR can survive treatment with $5 \mu \mathrm{M} 4-\mathrm{OHT}$. The levels of $\mathrm{NgBR}$ transcript and protein were determined by real-time PCR (Fig. 1e and f) and western blot analysis (Fig. 1g and h). The expression of NgBR was increased in both MCF-7-TamR (Fig. 1e, g and h) and T47D-TamR cells (Fig. 1f; Additional file 2: Figure S2) as compared to that in their parental cells. The alteration of other gene expression between MCF-7 and MCF7-TamR cells is shown in Fig. $1 \mathrm{~g}$ and $\mathrm{h}$. Consistent with many previous studies $[19,21,22]$, we also noted increased expression of EGFR, HER2, and survivin, and decreased expression of p53 and ER $\alpha$ in MCF-7-TamR (Fig. $1 \mathrm{~g}$ and h).

\section{$\mathrm{NgBR}$ knockdown attenuates the tamoxifen resistance}

To determine the contribution of increased NgBR expression to tamoxifen resistance, we knocked down the expression of $\mathrm{NgBR}$ with specific $\mathrm{NgBR}$ siRNA (siNgBR), which has been validated in our previous reports [18]. The effects of NgBR knockdown on cell apoptosis and necrosis of MCF-7-TamR (Fig. 2a and b) and T47D-TamR (Additional file 3: Figure $\mathrm{S} 3 \mathrm{~A}$ and $\mathrm{B}$ ) were determined by Annexin V/PI staining and flow cytometry. The results showed that NgBR knockdown increases the sensitivity of both MCF-7-TamR and T47D-TamR cells to 4-OHT. Compared to the non-treatment group, 4-OHT treatment alone did not induce significant death of tamoxifen-resistant cells. However, NgBR knockdown along with 4-OHT treatment significantly increased the percentage of cell death. Cell viability was determined by counting the number of negative trypan blue stained cells using a hemocytometer. Consistently, MCF-7-TamR and T47DTamR cells were resistant to 4-OHT treatment. However, NgBR knockdown restored the sensitivity of MCF-7-TamR (Fig. 2c) and T47D-TamR (Additional file 3: Figure S3C) to tamoxifen. The clonogenic survival assay further demonstrated that $\mathrm{NgBR}$ knockdown attenuates the colony formation capability of MCF-7-TamR (Fig. 2d and e) and T47D-TamR cells (Additional file 3: Figure S3D and E) under the condition of $5 \mu \mathrm{M} 4-\mathrm{OHT}$ treatment. These results demonstrated that $\mathrm{NgBR}$ knockdown increases the sensitivity of tamoxifen-resistant ER $\alpha$-positive breast cancer cells to tamoxifen.

As in our previous report [15], overexpression of $\mathrm{NgBR}$ in MCF-7 cells increased the membrane-associated H-Ras and K-Ras (Fig. 3a). Consequently, if we transfected plasmid DNA expressing human influenza hemagglutinin (HA) tagged NgBR (NgBR-HA) to MCF-7 cells, we appreciated that the overexpression of exogenous NgBR-HA increased the viability of MCF-7 cells treated with 4-OHT (Fig. 3b). Similarly, overexpression of HER2-HA also increased the resistance of MCF-7 cells to 4-OHT (Fig. 3b). However, NgBR knockdown attenuated the resistance of MCF-7 cells overexpressing HER2-HA (Fig. 3b). In MCF7-TamR cells, overexpression of NgBR-HA restored the resistance of NgBR knockdown MCF-7-TamR cells to tamoxifen (Fig. 3c). As shown in Fig. 3d, transfection of NgBR-HA plasmid DNA restored the expression of NgBR in MCF-7-TamR cells transfected with NgBR siRNA (siNgBR), which targets the 3 '-untranslated region (UTR) of $\mathrm{NgBR}$ as described in our previous publication [12]. It suggests that $\mathrm{NgBR}$ is one of driving forces for tamoxifen resistance. However, knockdown of Nogo-B does not affect the sensitivity of MCF-7-TamR to tamoxifen (Fig. 3c). The efficacy of Nogo-B siRNA was confirmed by western blot analysis (Fig. 3d).

\section{$\mathrm{NgBR}$ knockdown promotes apoptosis by regulating the expression of $\mathrm{p} 53$ and survivin}

Our previous study showed that NgBR deficiency in $E R \alpha$-positive breast cancer cells decreases the resistance to chemotherapy by increasing p53 and decreasing 

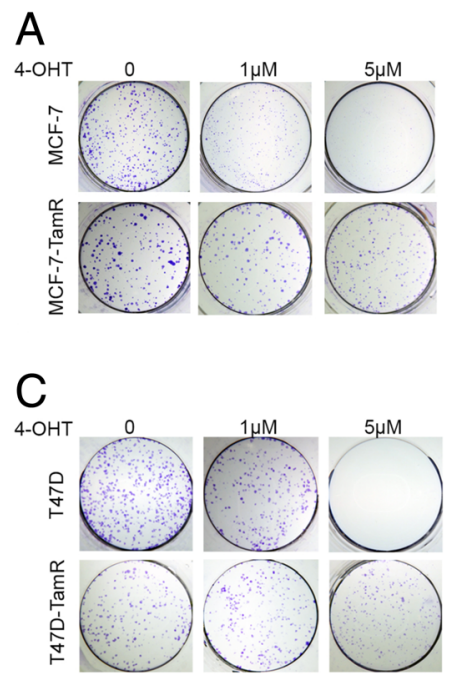

D
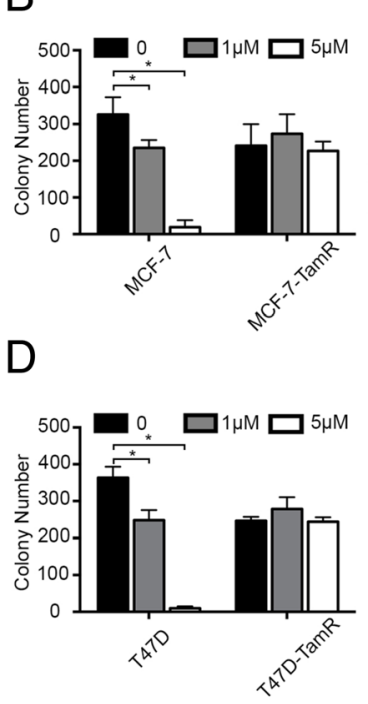

$\mathrm{F}$

\section{$\mathrm{E}$}
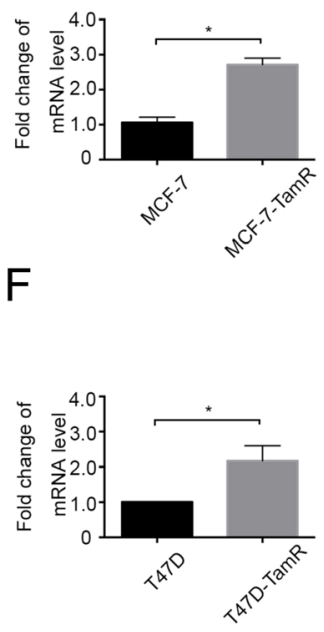

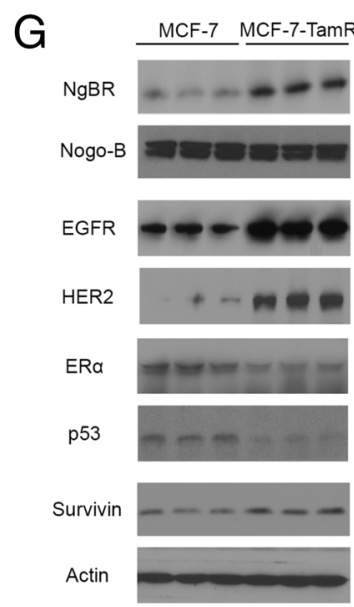

$\mathrm{H}$

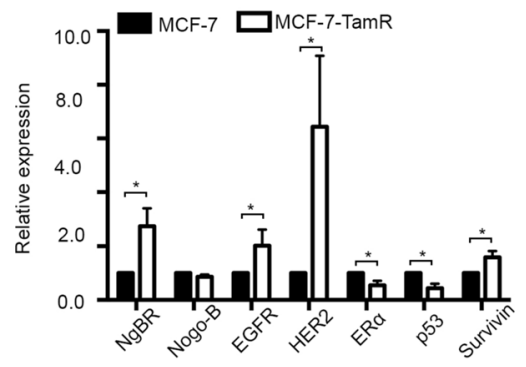

Fig. 1 Nogo-B receptor (NgBR) is highly expressed in the tamoxifen resistant MCF-7-TamR and T47D-TamR cells. a Colony formation assay was performed as described in "Methods". Wild-type MCF-7 and tamoxifen-resistant MCF-7-TamR cells were treated with different concentrations of 4-OHT (0, 1 and $5 \mu \mathrm{M})$. b Quantification of colony number presented in colony formation assays of MCF-7 and MCF-7-TamR cells. c Colony formation assay of wild-type T47D and tamoxifen-resistant T47D-TamR cells treated with different concentrations of 4-OHT (0, 1 and $5 \mu$ M). $\mathbf{d}$ Quantification of colony number in colony formation assays of T47D and T47D-TamR cells. e, $\mathbf{f}$ mRNA level of NgBR was increased in MCF-7-TamR and T47D-TamR cells as compared to wild-type MCF-7 and T47D cells, respectively. The relative amount of NgBR mRNA level was normalized to glyceraldehyde-3-phosphate dehydrogenase (GAPDH). g NgBR protein level was increased in MCF-7-TamR cells. Protein levels of Nogo-B, epidermal growth factor receptor (EGFR), human epidermal growth factor receptor 2 (HER2), estrogen receptor alpha (ERa), p53 and survivin in MCF-7 and MCF-7-TamR cells were determined using western blot analysis. $\mathbf{h}$ Quantitative analysis of protein levels using ImageJ and normalized to the housekeeping gene $\beta$-actin. Data are presented as fold changes in MCF-7-TamR cells compared to MCF-7 cells. The data are from three separate repeat experiments, and are presented as the mean \pm SD $\left({ }^{*} p<0.05, n=3\right)$

survivin [23]. To determine if and the extent to which NgBR is dependent on p53-mediated survivin expression to promote the resistance to tamoxifen, we examined the alteration of p53 and survivin expression in tamoxifen-resistant ER $\alpha$-positive breast cancer cells before and after NgBR depletion. As shown in Fig. 4a and b, NgBR depletion in MCF-7-TamR cells increased the expression of $\mathrm{p} 53$ but decreased the amount of survivin at the protein levels. Overexpression of NgBR-HA restored the expression pattern of p53 and survivin in
MCF-7-TamR cells transfected with siNgBR to levels similar to that in MCF-7-TamR cells transfected with control siRNA (Fig. 3d). When p53 was knocked down by siRNA, survivin was increased in MCF-7 cells (Fig. 4c and d). To determine if either p53 or survivin is involved in regulating the apoptosis of NgBR-deficient cells, we knocked down either p53 in MCF-7 cells or survivin in MCF-7-TamR cells using siRNA either targeting p53 (si-p53) or targeting survivin (si-survivin), respectively. As shown in Fig. 4e and f, knockdown of p53 increased 
A

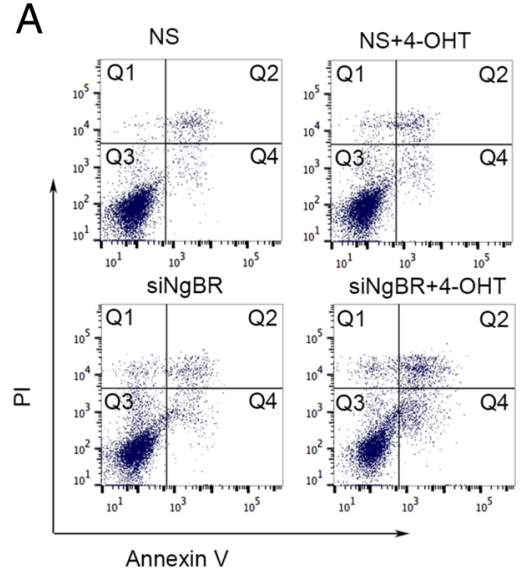

C

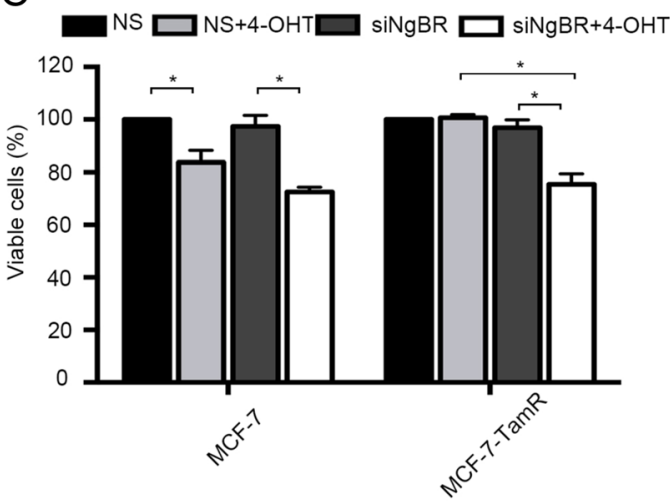

B

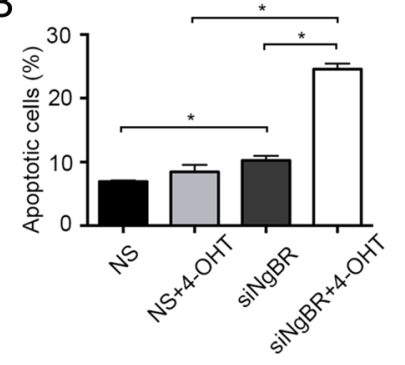

D

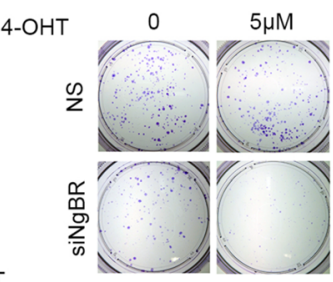

E

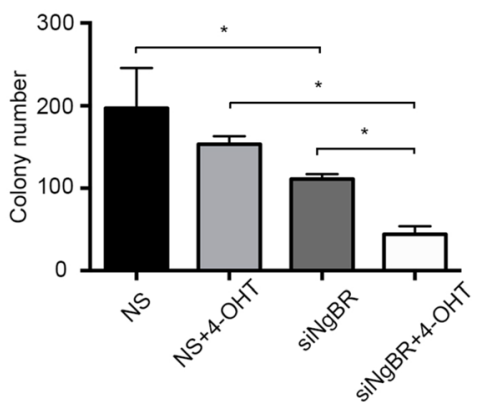

Fig. 2 Nogo-B receptor (NgBR) knockdown decreases the resistance of MCF-7-TamR cells to tamoxifen. a NgBR knockdown increases 4-OHTinduced apoptosis of MCF-7-TamR cells; 4-OHT, $5 \mu \mathrm{M}$. The apoptotic cells were detected by Annexin V-PI staining as described in "Methods". The total number of cells in the Q2 and Q4 quadrant were counted as apoptotic cells. b Percentages of apoptotic MCF-7-TamR cells are presented in the bar graph. c NgBR knockdown decreases cell viability of MCF-7-TamR cells treated with 4-OHT. Cell viability was determined using trypan blue staining. MCF-7-TamR cells were treated with 4-OHT (5 $\mu \mathrm{M})$ for $48 \mathrm{~h}$. The viable cell number in the non-silencing (NS) group is set as $100 \%$. d NgBR knockdown decreases the clonogenenicity of MCF-7-TamR cells treated with 4-OHT (5 $\mu$ M). Clonogenic survival assay was performed as described in "Methods". e Quantification of colony number in colony formation assays as described in Fig. 2d. The data were repeated in three separate experiments, and are presented as the mean $\pm \mathrm{SD}\left({ }^{*} p<0.05, n=3\right)$

the resistance of MCF-7 cells to 4-OHT, while survivin knockdown restored the sensitivity of MCF-7-TamR cells to 4-OHT (Fig. $4 \mathrm{~g}$ and h). Similar results were also observed in p53 knockdown T47D cells (Additional file 4: Figure S4A-D) and survivin knockdown T47D-TamR cells (Additional file 4: Figure S4E-F). These results demonstrated the contribution of increased p53 expression and decreased survivin expression that occurs in NgBR knockdown cells for restoring the sensitivity of ER $\alpha$-positive breast cancer cells to tamoxifen. The clonogenic survival assay further demonstrated that loss of p53 in NgBR knockdown MCF-7 cells attenuated the effects of $\mathrm{NgBR}$ deficiency on increased sensitivity of MCF-7 cells to tamoxifen (Fig. $4 \mathrm{i}$ and j). In addition, knockdown of both H-Ras and K-Ras in MCF-7-TamR cells also resulted in the increased amount of p53 and decreased amount of survivin (Fig. 5a). Overexpression of either NgBR-HA or HER2-HA in MCF-7 cells decreased the protein levels of $\mathrm{p} 53$ and $E R \alpha$ but increased the protein level of survivin (Fig. 5b). Interestingly, knockdown of NgBR in MCF-7 cells overexpressing HER2-HA restored the protein levels of p53, ER $\alpha$ and survivin levels to those occurring in control MCF-7 cells transfected with empty vector (Fig. 5b).

\section{NgBR knockdown diminished EGF-stimulated phosphorylation of ERa}

Phosphorylation of ER $\alpha$ serine 118 (S118) residue has been reported to be involved in resistance to tamoxifen $[24,25]$. To examine the involvement of NgBR in regulating EGF-stimulated phosphorylation of ER $\alpha$ S118, we treated MCF-7-TamR cells with $100 \mathrm{ng} / \mathrm{mL}$ EGF for 


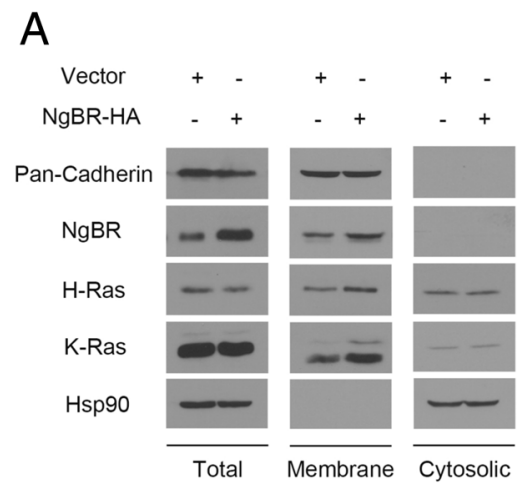

C

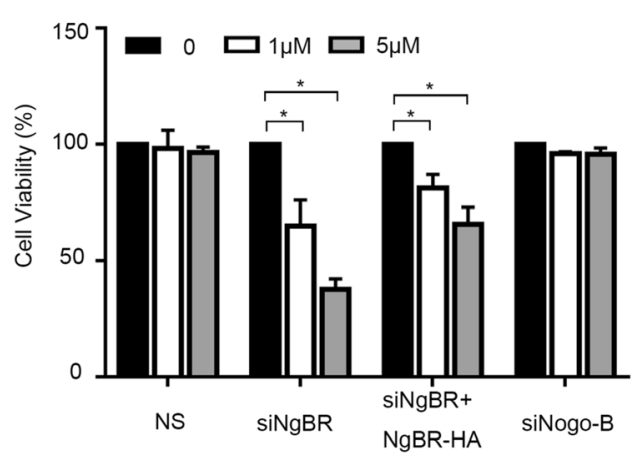

B

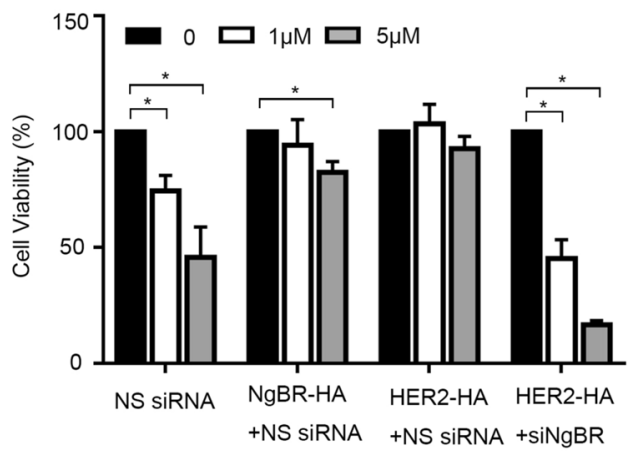

D

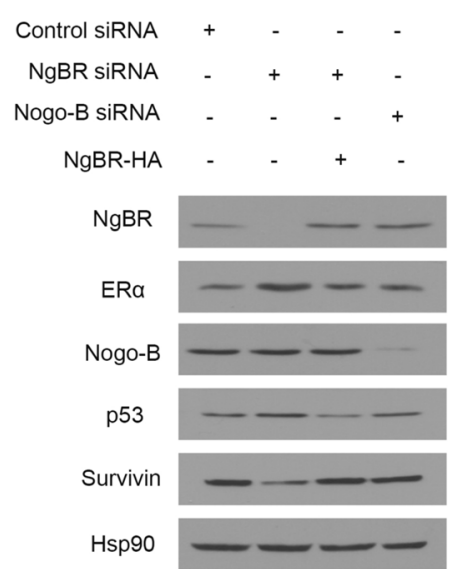

Fig. 3 Overexpression of Nogo-B receptor (NgBR) increases the resistance of MCF-7 cells to tamoxifen. a Overexpression of NgBR in MCF-7 cells increases the membrane-associated H-Ras and K-Ras. The plasma membrane proteins were isolated by the ultracentrifugation method. Protein levels of pan-cadherin, NgBR, H-Ras, K-Ras and Hsp90 in MCF-7 cells were determined using western blot analysis. b Viability of MCF-7 cells treated with 4-OHT (0, 1 or $5 \mu \mathrm{M})$ was determined using CCK-8 assay. Overexpression of either human influenza hemagglutinin (HA)-tagged NgBR or HER2 in MCF-7 cells decreases their sensitivity to 4-OHT. Knockdown NgBR in MCF-7 cells restores the sensitivity of MCF-7 cells overexpressing HER2-HA to 4-OHT. The number of viable cells in the untreated group is referred as $100 \%\left({ }^{*} p<0.05, n=3\right)$. c Viability of MCF-7-TamR cells treated with 4-OHT (0, 1 or $5 \mu \mathrm{M})$ was determined using CCK-8 assay. Knockdown of NgBR in MCF-7-TamR cells increases their sensitivity to 4-OHT. Overexpression of NgBR decreases the sensitivity of NgBR-knockdown MCF-7-TamR cells to 4-OHT. Knockdown of Nogo-B in MCF-7-TamR cells does not affect their sensitivity to $4-\mathrm{OHT}$. The number of viable cells in the untreated group is referred to as $100 \%\left({ }^{*} p<0.05, n=3\right)$. $\mathbf{d}$ NgBR regulates the expression of ERa, p53 and survivin independent of its ligand Nogo-B. MCF-7-TamR cells were transfected with control siRNA or NgBR siRNAs targeting either NgBR or Nogo-B. In MCF-7-TamR cells transfected with siRNA targeting the untranslated region of NgBR, NgBR expression was restored by the transfection of NgBR-HA plasmid DNA

5 min, which is the peak of the phosphorylation signal in response to EGF stimulation. As shown in Fig. 6a and b, EGF treatment increased the phosphorylation of AKT, ERK and MDM2 in MCF-7-TamR cells treated with control non-silencing (NS) siRNA. NgBR knockdown attenuated EGF-stimulated phosphorylation of AKT, ERK and MDM2. But NgBR knockdown did not affect the total protein levels of AKT, ERK and MDM2 or the phosphorylation of EGFR (Fig. 6a). EGF treatment not only activated the downstream signaling of the EGF pathway, but also increased the phosphorylation of ER $\alpha$ (S118), which is in accordance with previous studies [10, 26]. NgBR knockdown attenuated EGF-stimulated phosphorylation of ER $\alpha$. The inhibitory effects of NgBR knockdown on EGF-stimulated phosphorylation of ER $\alpha$ were also noted in T47D-TamR cells (Additional file 5: Figure S5).

To further investigate the underlying mechanism by which NgBR regulates the expression of p53 and survivin, we stimulated MCF-7-TamR cells with EGF $(100 \mathrm{ng} / \mathrm{mL})$ for $12 \mathrm{~h}$. EGF treatment increased the protein level of survivin, and NgBR knockdown attenuated the protein level of survivin in EGF-treated cells (Fig. $6 \mathrm{c}$ and d). To elucidate the roles of $\mathrm{NgBR}$ in regulating the EGF-mediated pathway, we used glutathione (GST)-tagged Ras-binding domain of Raf (RBD) to pull down activated Ras as described in our previous publication [15]. As shown in Fig. 6e, EGF stimulation for 5 min not only induced the 


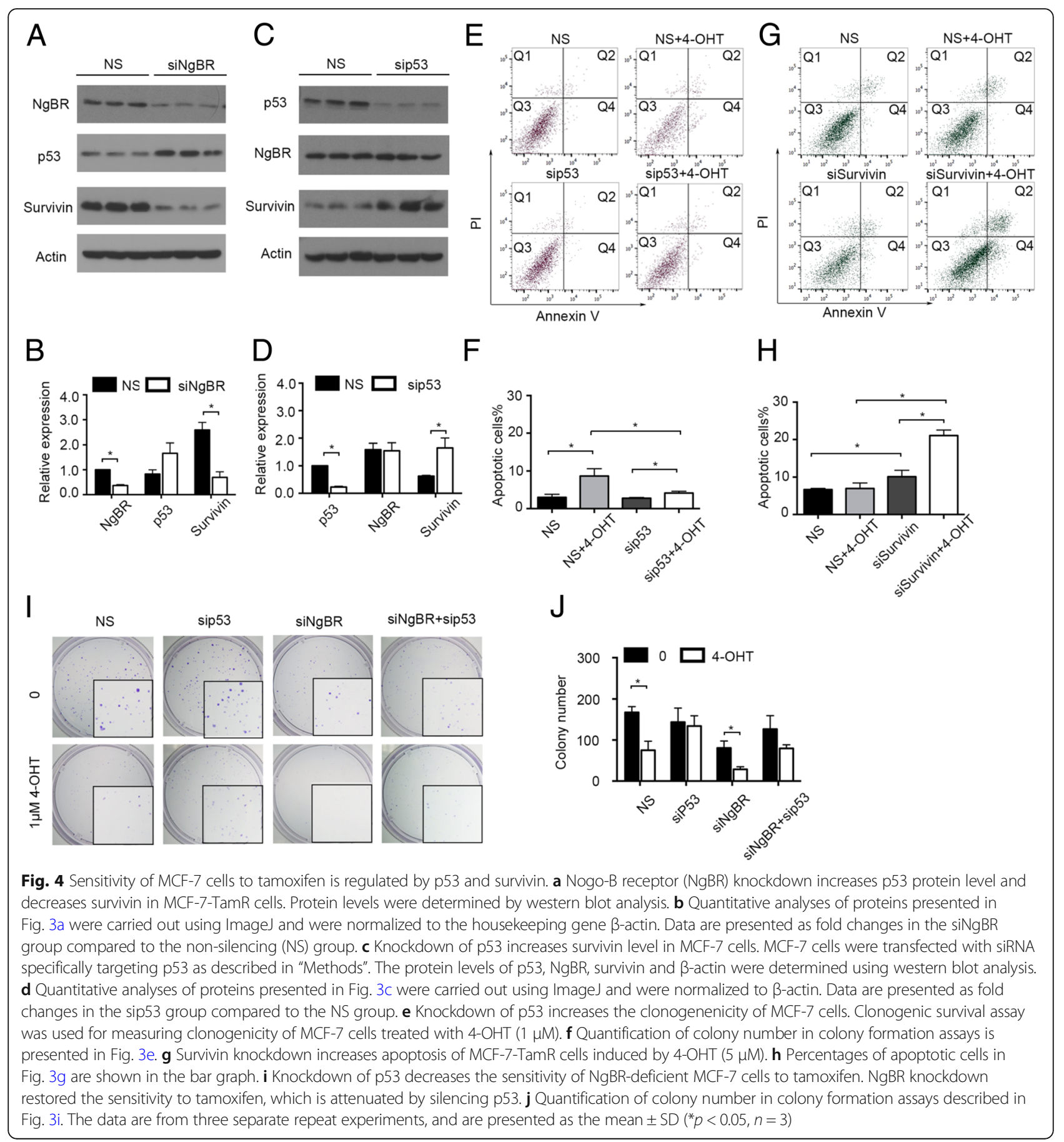

activation of H-Ras and K-Ras in MCF-7-TamR cells, but also increased the amount of $\mathrm{NgBR}$ in the complex of activated $\mathrm{K}$-Ras and $\mathrm{H}$-Ras. It indicates that EGF stimulation increases the association between $\mathrm{NgBR}$ and activated Ras. Consistent with our previous reports [15], NgBR knockdown also attenuated the EGF-stimulated Ras activation in MCF-7-TamR cells. These results (Fig. 6) suggest that NgBR-mediated Ras activation may contribute to EGF-stimulated phosphorylation of ER $\alpha$.

\section{$\mathrm{NgBR}$ expression is associated with survivin and poor survival in patients with breast cancer}

Our previous publication showed that expression of $\mathrm{NgBR}$ is much higher in ER $\alpha$-positive breast cancer tissues than in normal breast tissues, and that $\mathrm{NgBR}$ is also highly associated with survivin expression [16]. To further confirm the relationship between survivin and $\mathrm{NgBR}$, we performed immunohistochemistry (IHC) staining to examine expression in 22 samples of breast 
A
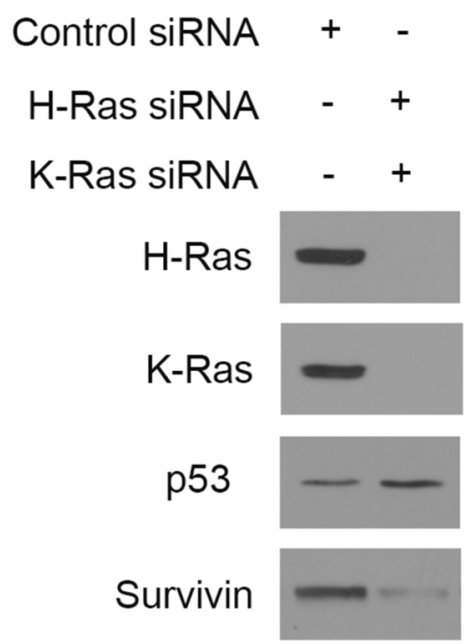

$\mathrm{NgBR}$

Hsp90
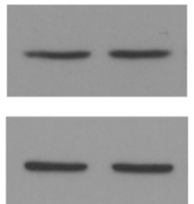

B

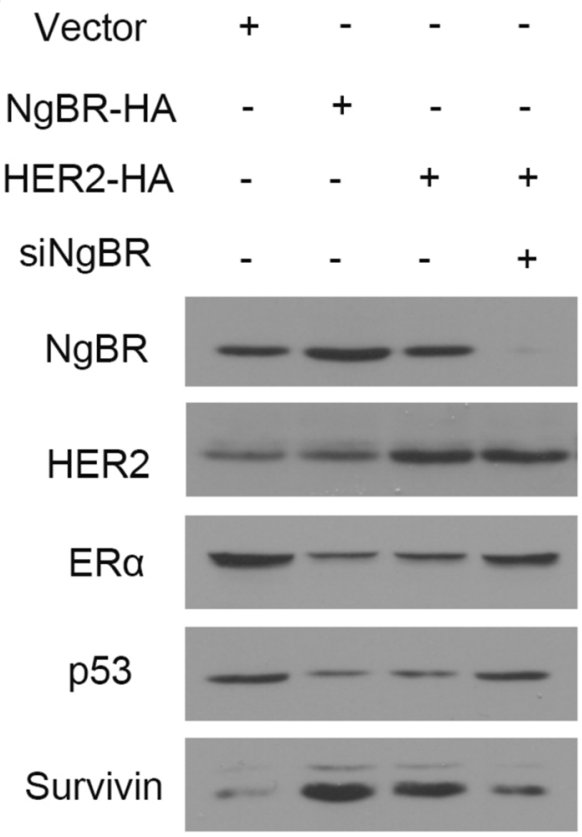

AKT

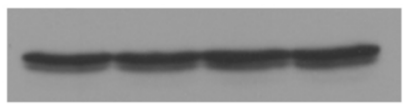

ERK

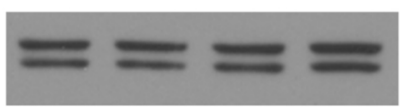

Hsp90

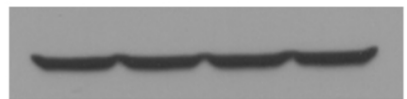

Fig. 5 Nogo-B receptor (NgBR) regulates protein levels of p53 and survivin through Ras-mediated pathways. a Knockdown of both H-Ras and KRas increases the protein levels of p53 and decreases the protein level of survivin. Control siRNA and siRNAs targeting either H-Ras or K-Ras siRNA were transfected into MCF-7-TamR cells. b Overexpression of either NgBR-HA or HER2-HA in MCF-7 cells decreases the protein levels of p53, ERa, and increases the protein level of survivin. Knockdown NgBR in MCF-7 cells overexpressing HER2-HA restores the protein levels of p53, ERa and survivin to levels similar to those in control MCF-7 cells transfected with empty plasmid DNA vector. Change in NgBR or human epidermal growth factor receptor 2 (HER2) has no effects on the total protein levels of AKT and extracellular signal-related kinase (ERK)

cancer tissue. The basic characteristics of the tissue samples are shown in Table 1, and the results of the quantitative analysis of IHC staining was shown in Table 2. As shown in Fig. 7a, patients with high expression of NgBR also have high expression of survivin. In patients with negative or weak expression of $\mathrm{NgBR}$, the expression of survivin was also low. The association between NgBR and survivin was statistically significant (Table 2). The association between NgBR and clinical outcomes in patients with breast cancer was determined using the public Kaplan-Meier Plot database. Kaplan-Meier analysis revealed that high expression of NgBR was associated with poor RFS in patients with ER $\alpha$-positive breast cancer $(n=755)$ and in patients receiving endocrine therapy $(n=335)$ (Fig. 7b; Additional files 6, 7, 8 and 9). Consistently, high expression of survivin was also associated with poor RFS in patients with ER $\alpha$-positive breast cancer $(n=2046)$ and in patients receiving endocrine therapy $(n=928) \quad$ (Fig. 7c). The association between higher NgBR expression and poor RFS was further confirmed in the GSE6532 dataset $(n=343)$ (Additional file 10: Figure S6; Additional file 11).

\section{Discussion}

As previously confirmed, NgBR is highly expressed in ER $\alpha$-positive breast cancer [16], and promotes epithelialmesenchymal transition of breast tumor cells [18]. However, the underlying mechanism by which NgBR enhances the acquired resistance of ER $\alpha$-positive breast cancer to tamoxifen has not been elucidated. In this study, we found that NgBR expression is increased in tamoxifen-resistant breast cancer cell lines (Fig. 1). High expression of $\mathrm{NgBR}$ was associated with poor RFS in patients with $E R \alpha$-positive breast cancer and in patients 


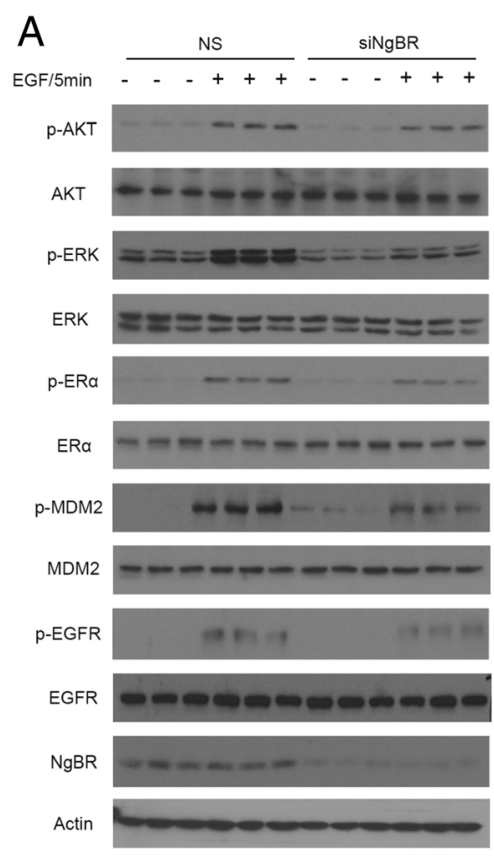

B

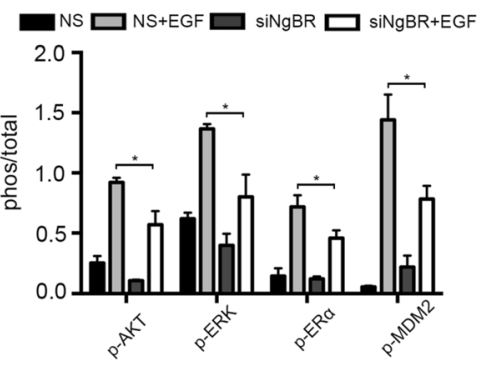

C

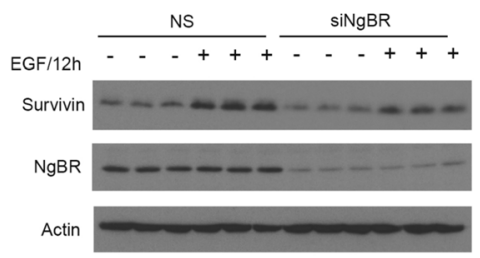

D

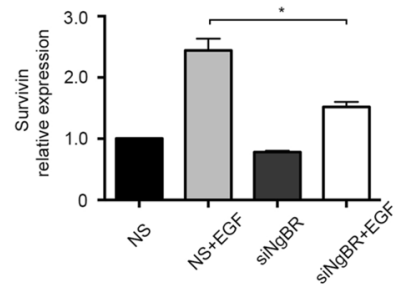

$\mathrm{E}$

EGF/5min $\frac{N S}{-+} \frac{\text { siNgBR }}{-+}+\frac{\text { NS }}{-+} \frac{\text { siNgBR }}{-+}$
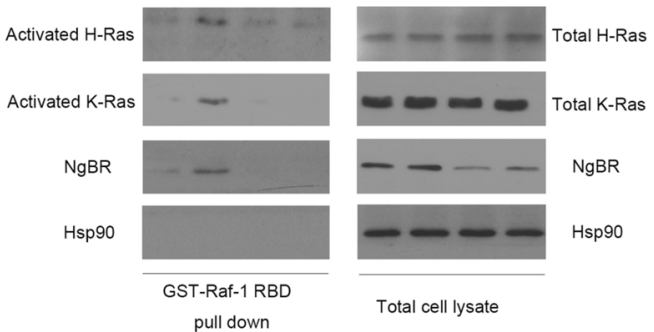

Fig. 6 Nogo-B receptor (NgBR) knockdown attenuates epidermal growth factor receptor (EGF)-stimulated signaling and estrogen receptor alpha (ERa) phosphorylation in MCF-7-TamR cells. a MCF-7-TamR cells were transfected with siNgBR and treated with EGF (100 ng/mL) for 5 min. Downstream signaling of the EGF pathway was determined using western blot assay. b Quantitative analysis of phosphorylated proteins presented in Fig. 4b were carried out using ImageJ and were normalized to total proteins. c MCF-7-TamR cells were transfected with siNgBR and treated with $100 \mathrm{ng} / \mathrm{mL}$ EGF for $12 \mathrm{~h}$. Survivin protein levels were determined using western blot analysis. d Quantitative analysis of survivin protein levels presented in Fig. 4c were carried out using ImageJ and were normalized to $\beta$-actin. e NgBR is required for the EGF-stimulated activation of H-Ras and K-Ras in MCF-7-TamR cells. MCF-7-TamR cells were transfected with siNgBR and stimulated with $100 \mathrm{ng} / \mathrm{mL}$ EGF for $5 \mathrm{~min}$. The complex of activated Ras (GTP-loaded Ras) was precipitated from total cell lysates using GST-RBD beads. Protein levels were detected by western blotting. Both Ras and NgBR were detected in the complexes precipitated by the Raf-pull-down method. The data are from three separate repeat experiments and are presented as the mean \pm SD $\left({ }^{*} p<0.05, n=3\right)$

receiving endocrine therapy (Fig. 5b). NgBR knockdown decreased EGF-induced expression of survivin (Fig. 4c and d) and phosphorylation of ER $\alpha$ (Fig. 4a). Consequently, the results of cell viability and apoptosis assays clearly demonstrate that NgBR knockdown attenuates resistance to tamoxifen (Fig. 2). Our study elucidated the important roles of NgBR in promoting the acquired resistance of ER $\alpha$-positive breast cancer to tamoxifen.

For patients with ER $\alpha$ positive breast cancer, treatment mainly focuses on reducing estrogen levels or blocking the ER $\alpha$ signaling pathway. Aromatase inhibitors (AIs), such as anastrozole [27], are estrogen synthesis inhibitors. Fulvestrant is a selective ER $\alpha$ downregulator [28]. Tamoxifen, also known as a selective estrogen receptor modulator, blocks the activity of estrogen by binding to ER $\alpha$ [29] and suppressing the classical ERE regulated genes [30]. However, $30 \%$ of patients still gain resistance to tamoxifen [31]. Many studies have elucidated potential mechanisms of tamoxifen resistance, but these are still unclear due to many unidentified factors [32]. In this study, we demonstrated that $\mathrm{NgBR}$, which is upregulated in tamoxifenresistant breast cancer cells, is a potential factor contributing to tamoxifen resistance. Our data demonstrated that NgBR knockdown restores the sensitivity of tamoxifenresistant breast cancer cells to tamoxifen (Fig. 2). Our result indicates $\mathrm{NgBR}$ is a potential therapeutic target for 
Table 1 Demographic and clinical characteristics of study population

\begin{tabular}{lll}
\hline & Variables & Value \\
\hline ER & $1+$ & $1(0.05)$ \\
& $2+$ & $9(0.41)$ \\
PR & $3+$ & $12(0.54)$ \\
& $1+$ & $4(0.18)$ \\
& $2+$ & $5(0.23)$ \\
HER2 & $3+$ & $13(0.59)$ \\
& - & $17(0.77)$ \\
& $1+$ & $2(0.09)$ \\
& $2+$ & $2(0.09)$ \\
& $3+$ & $1(0.05)$ \\
\hline
\end{tabular}

Abbreviations: $E R$ estrogen receptor, $P R$ progesterone receptor, HER2 human epidermal growth factor receptor 2

attenuating tamoxifen resistance. Unlike NgBR, Nogo-B protein levels do not increase in either MCF-7-TamR cells (Fig. 1g) or T47D-TamR cells (Additional file 2: Figure S2). Although NgBR was identified as a specific receptor for ligand Nogo-B, knockdown of Nogo-B does not affect the sensitivity of MCF-7-TamR cells to tamoxifen (Fig. 3c). If and the extent to which Nogo-B facilitates the NgBR-mediated Ras-signaling pathway needs further investigation.

According to previous studies, increased EGFR expression in tamoxifen-resistant cells contributes to the acquired resistance to tamoxifen [10, 21]. Consistent with previous reports, EGF stimulation also activated the phosphorylation of ER $\alpha$ [33]. Our recent report demonstrated that NgBR binds the farnesylated Ras and promotes Ras plasma membrane translocation [15]. NgBR-mediated accumulation of plasma membrane-associated Ras enhances EGF signaling [15]. As shown in Fig. 3a, NgBR overexpression in MCF-7 cells also increased membraneassociated H-Ras and K-Ras and resulted in increased resistance to tamoxifen (Fig. 3b). Similarly, increased expression of NgBR in tamoxifen-resistant breast cancer cells also enhances EGF-stimulated Ras activation and phosphorylation of AKT and ERK. NgBR knockdown diminishes the EGF-stimulated Ras activation and EGFRmediated signaling (Fig. 6). Activation of these pathways leads to Akt-dependent phosphorylation of MDM2 [34], which downregulates cellular levels of p53 and decreases

Table 2 Correlation analysis of survivin and NgBR

\begin{tabular}{|c|c|c|c|c|}
\hline & \multicolumn{2}{|l|}{ Survivin } & \multirow[t]{2}{*}{ Number } & \multirow[t]{2}{*}{$p$} \\
\hline & Low & High & & \\
\hline \multicolumn{5}{|l|}{$\mathrm{NgBR}$} \\
\hline Low & 5 (71.4\%) & $2(28.6 \%)$ & 7 (100\%) & 0.014 \\
\hline High & $2(13.3 \%)$ & 13 (86.7\%) & 15 (100\%) & \\
\hline$n$ & 7 (31.8\%) & $15(68.2 \%)$ & $22(100 \%)$ & \\
\hline
\end{tabular}

NgBR Nogo-B receptor p53 transcriptional activity [35]. Our previous report also demonstrated that $\mathrm{NgBR}$ promotes the ubiquitination of p53 in human hepatocellular carcinoma via Akt and MDM2 phosphorylation signaling [36]. Here, our data demonstrated the inverse expression patterns between p53 and NgBR in breast cancer cells (Fig. 4). The protein levels of p53 decrease in tamoxifen-resistant breast cancer cells along with increased expression of survivin and NgBR (Fig. 1g; Additional file 2: Figure S2A). Knockdown of either NgBR (Fig. 3d) or H-Ras/K-Ras (Fig. 5a) in MCF-7-TamR cells increased p53 and deceased survivin protein levels. As a tumor repressor gene, p53 is found to be mutated in many cancers [37] and promotes apoptosis in breast cancers [38]. It has been shown that p53 represses survivin expression at the transcriptional level [39]. Our data (Fig. 4c and d; Additional file 4: Figure S4A and B) also demonstrated that knockdown of p53 in breast cancer cells can induce the expression of survivin, which predicts a poor response to endocrine therapy [40]. In this study, we confirmed the effects of p53 on inducing tamoxifen resistance in parental MCF-7 and T47D cells, and survivin knockdown restored the sensitivity to 4-OHT in MCF-7-TamR (Fig. 4) and T47D-TamR cells (Additional file 4: Figure S4).

Ras is a well-known oncogene that has been shown to cause tumorigenesis and drug resistance by activating downstream kinases such as phosphatidylinositol-3-OH kinase (PI-3 K)/Akt and Raf-1 kinase/ERK [41-45]. Although Ras mutations rarely occur in breast cancer (less than 10\%) [46], oncogenic Ras can contribute to the tumorigenic and invasive potential of breast epithelial cells [46]. Therefore, upregulation of normal Ras activity by RTKs, such as the EGFR and insulin growth factor receptor (IGF1-R), has been shown in ER $\alpha$ positive breast cancer [47-49]. The classic mechanism of E2 action is mediated by the nuclear ER $\alpha$ that regulates transcription of target genes containing the consensus ERE in their promoter region [47-49]. In addition, E2 can also exert its action through membrane $E R \alpha(m E R \alpha)$ in conjugation with the signaling complex including EGFR, IGF-1R, adaptor protein Shc/Grb2 and RasGEFs (such as SOS1 and RasGEF3) to activate Src/Ras-dependent activation of the Raf1-MARK/PI3K-Akt pathways [47-49]. This pathway promotes estrogen-dependent tumor resistance [50]. Our recent publication demonstrated that NgBR binds farnesylated Ras and is required for keeping Ras at the plasma membrane [15]. Therefore, NgBR is essential for the Ras-mediated signaling pathway [15]. Previous reports have shown that EGF induces the phosphorylation of ER $\alpha$ at the serine 118 residue [51], which is the confirmed signal involved in ER $\alpha$-mediated resistance to endocrine therapy [25]. In this study, we demonstrated that NgBR knockdown impairs EGF-stimulated phosphorylation of $\mathrm{ER} \alpha$ (Fig. 4a; Additional file 10: Figure S6A) but also 

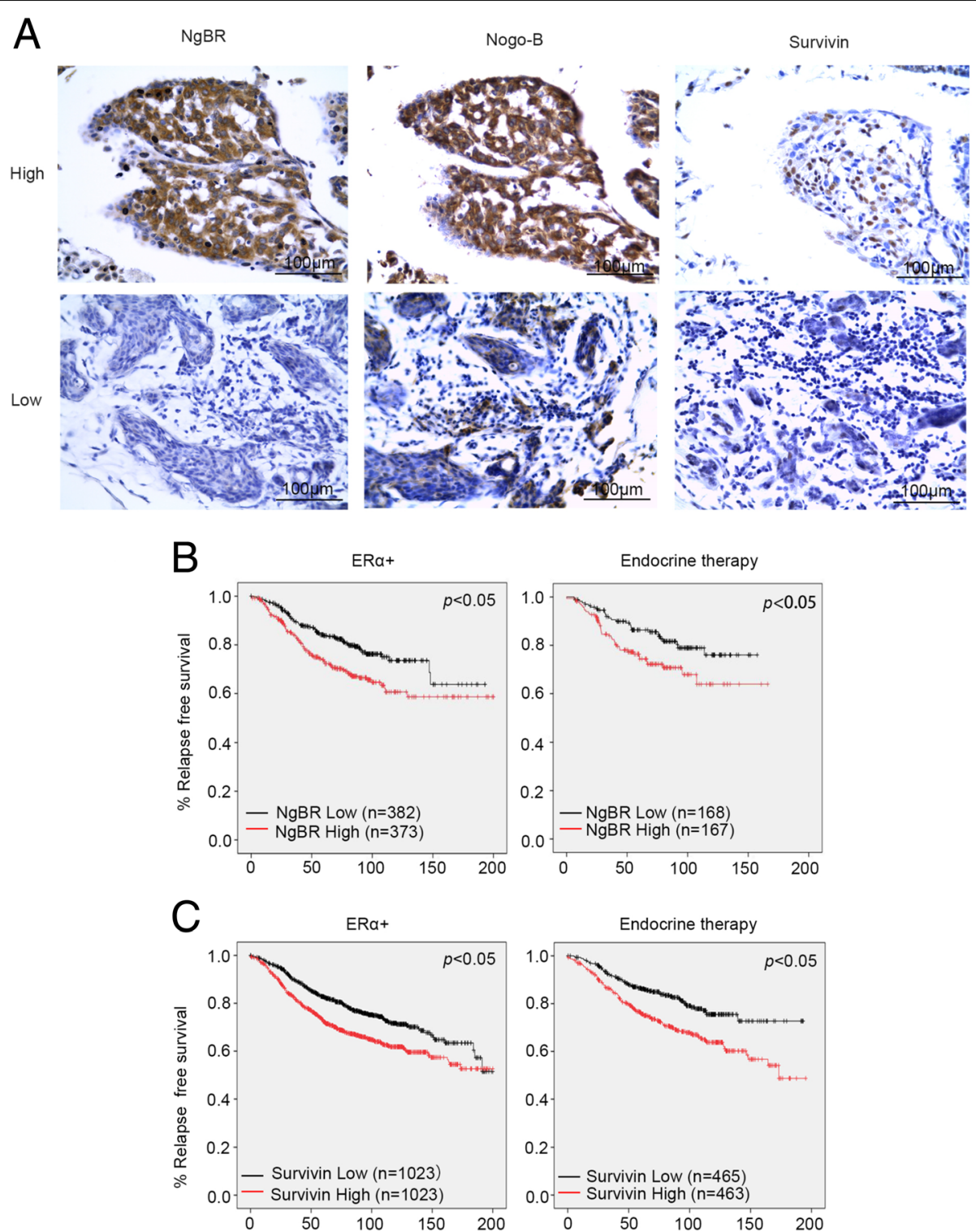

Fig. 7 Higher expression of Nogo-B receptor (NgBR) is associated with poor outcome in patients with estrogen receptor alpha (ERa) positive breast cancer. a Immunohistocheical (IHC) staining of NgBR, Nogo-B and survivin in 22 samples of breast cancer tissue. Images were taken using an Olympus microscope with $\times 20$ lens. Scale bar $100 \mu \mathrm{m}$. b Relapse-free survival (RFS) in patients with ERa-positive breast cancer or endocrine therapy-treated patients. NgBR (NUS1) mRNA expression data were retrieved from a gene-expression profiling dataset (225071_x from Kaplan-Meier Plot database) of 755 cases of ERa-positive breast cancer and 335 patients with ERa-positive breast cancer treated with endocrine therapy. Kaplan-Meier analysis revealed significantly reduced RFS $(p<0.05)$ in 373 patients with ERa-positive breast cancer with high NgBR expression in tumors as compared to 382 patients with low NgBR expression in tumors. Similarly, RFS in patients with ERa-positive breast cancer treated with endocrine therapy is significantly decreased in 167 patients with high NgBR expression in tumors as compared to 168 patients with low NgBR expression in tumors ( $p<0.05)$. c RFS in patients with ERa-positive breast cancer or endocrine therapy-treated patients. Survivin (BIRC5) mRNA expression data were retrieved from a geneexpression profiling dataset (202094_x from Kaplan-Meier Plot database) of 2046 cases of ERa-positive breast cancer and 928 patients with ERapositive breast cancer treated with endocrine therapy. Kaplan-Meier analysis revealed significantly reduced RFS $(p<0.05)$ in 1023 patients with ERapositive breast cancer with high survivin expression in tumors as compared to 1023 patients with low survivin expression in tumors. Similarly, RFS in patients with ERa-positive breast cancer treated with endocrine therapy is significantly decreased in 463 patients with high survivin expression in tumors as compared to 465 patients with low NgBR expression in tumors $(p<0.05)$

attenuates resistance to tamoxifen (Fig. 2; Additional file 3: Figure S3). This finding indicates that NgBR is a potential therapeutic target for blocking concurrent endocrine-resistant signaling. However, we need further investigation to determine synergetic roles of $\mathrm{NgBR}$ in coordinating with other growth factor receptors, such as insulin-like growth factor 1 receptor (IGF1-R) and $\mathrm{mER} \alpha$, to promote the acquired resistance to tamoxifen. 


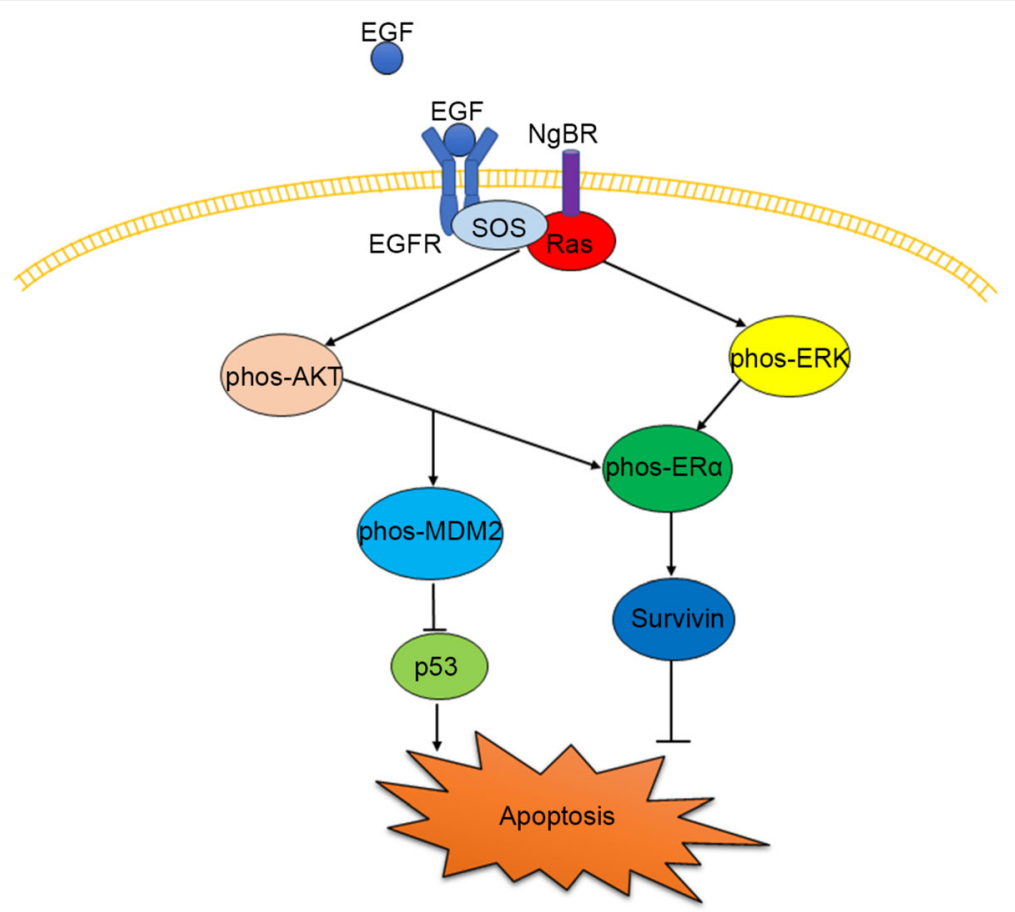

Fig. 8 Nogo-B receptor (NgBR) is required for epidermal growth factor (EGF)-acquired resistance to tamoxifen in patients with estrogen receptor alpha (ERa)-positive breast cancer. EGF binds to EGF receptor (EGFR) and recruits SOS1, an activator for Ras, to the plasma membrane. NgBR promotes the translocation of Ras to the plasma membrane and enhances EGF signaling, such as phosphorylation of Akt and extracellular signalrelated kinase (ERK). Phosphorylated Akt further induces the phosphorylation of MDM2, which promotes p53 ubiquitination leading to its degradation and attenuates p53-mediated apoptosis. Phosphorylation of Akt and ERK also induce the phosphorylation of ERa, which induces the expression of survivin, an apoptosis inhibitor

Except for the contribution of growth factor receptor signaling pathways to tamoxifen resistance $[9,10]$, decreased $E R \alpha$ expression [7, 52] and increased human epidermal growth factor receptor 2 (HER2) $[8,53]$ are more likely to be attributed to tamoxifen resistance. Previous studies by others showed that hyperactivation of Raf kinase induces the loss of ER $\alpha$ in ER $\alpha$-positive breast cancer cells [54] and inhibition of mitogen-activated protein kinase (MAPK) activity induced the expression of ER $\alpha$ in ER $\alpha$-negative breast cancer cells [55]. As shown in Fig. 1g, ER $\alpha$ decreased and HER2 increased in MCF-7-TamR cells as compared to parental MCF-7 cells. Overexpression of either NgBR-HA or HER2-HA in MCF-7 cells decreased the protein level of ER $\alpha$ (Fig. 5b). Interestingly, NgBR knockdown in either MCF-7-TamR cells (Fig. 3d and c) or MCF-7 cells overexpressing HER2-HA (Figs. 5b and $3 \mathrm{~b}$ ) restored the protein level of ER $\alpha$ to a level similar to that in control cells as well as sensitivity to tamoxifen. The contribution of NgBRregulated ER $\alpha$ expression to tamoxifen resistance needs further investigation in our future studies.

\section{Conclusions}

In summary, our results demonstrate that increased expression of $\mathrm{NgBR}$ in tamoxifen-resistant breast cancer cells promotes EGF signaling by increasing phosphorylation of AKT/ERK and MDM2, which attenuates the expression of p53 and increases the expression of survivin, which may lead to acquired resistance to tamoxifen as shown in Fig. 8. Higher levels of NgBR in patients with ER $\alpha$-positive breast cancer are associated with poor RFS outcomes in patients with breast cancer because they lead to easier acquisition of tamoxifen resistance. Our data suggest that $\mathrm{NgBR}$ is a potential therapeutic target for restoring the sensitivity of tamoxifen-resistant breast cancer cells to conventional endocrine therapy.

\section{Additional files}

Additional file 1: Figure S1. MCF-7-TamR and T47D-TamR cells are resistant to 4-OHT. The 4-OHT resistant phenotype was confirmed using the CCK8 cell viability assay. (A) Cell viability was analyzed in MCF-7 and MCF-7-TamR cells treated with $1 \mu \mathrm{M} 4 \mathrm{OHT}$ for different time periods ( 0,1 day, 3 days and 5 days). (B) Cell viability was analyzed in T47D and T47D-TamR cells treated with $1 \mu \mathrm{M}$ 4OHT for different time periods $(0,1$ day, 3 days and 5 days). The OD value of untreated cells is referred to as $100 \%$. The results show the average percentage of OD value as compared to untreated cells. The data are from in three separate repeated experiments, and are presented as the mean $\pm \mathrm{SD}\left({ }^{*} p<0.05, n=3\right)$. (PDF $153 \mathrm{~kb}$ )

Additional file 2: Figure S2. NgBR is highly expressed in the tamoxifenresistant T47D-TamR cells. (A) NgBR level was increased in T47D-TamR 
cells. Protein levels of Nogo-B, ERa, p53 and survivin in T47D and T47DTamR cells were determined using western blot analysis. (B) Quantitative analysis of proteins presented in Additional file 2: Figure S2A was carried out using ImageJ and normalized to $\beta$-actin. Data are presented as fold changes of T47D-TamR compared to the T47D cells. The data are from three separate repeated experiments and are presented as the mean \pm SD $\left({ }^{*} p<0.05, n=3\right)$. (PDF $\left.155 \mathrm{~kb}\right)$

Additional file 3: Figure S3. NgBR decreases the resistance of T47DTamR to tamoxifen. (A) NgBR knockdown increases apoptosis of T47DTamR cells induced by 4-OHT (5 $\mu \mathrm{M})$. The apoptotic cells were detected by Annexin V-PI staining. The total number of cells in the Q2 and Q4 quadrant was regarded as apoptotic cells. (B) Percentages of apoptotic cells are shown in the bar graph. (C) NgBR knockdown decreases the viability of T47D-TamR cells. Cell viability was determined using trypan blue staining of T47D-TamR cells treated with $5 \mu \mathrm{M} 4-\mathrm{OHT}$ for $48 \mathrm{~h}$. The viable cell number of the NS group is referred to as 100\%. (D) NgBR knockdown decreases the clonogenenicity of T47D-TamR cells. The clonogenic survival assay was used for measuring clonogenicity of T47DTamR cells treated with 4-OHT (5 $\mu \mathrm{M})$. (E) Quantification of colony number in colony formation assays presented in Additional file 3: Figure S3D. The data are from three separate repeated experiments and are presented as the mean \pm SD $\left({ }^{*} p<0.05, n=3\right)$. (PDF $296 \mathrm{~kb}$ )

Additional file 4: Figure S4. Sensitivity of T47D cells to tamoxifen is regulated by $\mathrm{p} 53$ and survivin (A) Knockdown of $\mathrm{p} 53$ increases survivin level in T47D cells. T47D cells were transfected with siRNA specifically targeting p53 as described in "Methods". The protein levels of p53, NgBR, survivin and $B$-actin were determined using western blot analysis. (B) Quantitative analysis of proteins presented in Additional file 5: Figure S5A were carried out using ImageJ and were normalized to $\beta$-actin. Data are presented as fold changes in the si-p53 group compared to the NS group. (C) Knockdown of p53 decreases apoptosis of T47D cells induced by 4-OHT (1 $\mu \mathrm{M})$. (D) Percentages of apoptotic cells in Additional file 5: Figure S5C are shown in the bar graph. (E) Survivin knockdown increases apoptosis of T47D-TamR cells induced by $4-\mathrm{OHT}(5 \mu \mathrm{M})$. (F) Percentages of apoptotic cells in Additional file 5: Figure S5E are shown in the bar graph. (PDF $292 \mathrm{~kb}$ )

Additional file 5: Figure S5. NgBR knockdown attenuated EGFstimulated signaling and ERa phosphorylation in T47D-TamR cells. (A) T47D-TamR cells were transfected with siNgBR and treated with EGF $(100 \mathrm{ng} / \mathrm{mL})$ for $5 \mathrm{~min}$. Downstream signaling of the EGF pathway was determined using western blot assay. (B) Quantitative analysis of phosphorylated proteins presented in Additional file 10: Figure S6A were carried out using ImageJ and were normalized to total proteins. The data are from three separate repeated experiments and are presented as the mean \pm SD $\left({ }^{*} p<0.05, n=3\right)$. (PDF $\left.310 \mathrm{~kb}\right)$

Additional file 6: NgBR (NUS1) mRNA expression data were retrieved from a gene-expression profiling dataset (225071_x from Kaplan-Meier Plot database) of 755 patients with ERa-positive breast cancer and 335 patients with ERa-positive breast cancer treated with endocrine therapy. Kaplan-Meier analysis revealed significantly reduced relapse-free survival (RFS) $(p<0.05)$ in 373 patients with ERa positive breast cancer with high $\mathrm{NgBR}$ expression in tumors as compared to 382 patients with low NgBR expression in tumors. (XLSX $42 \mathrm{~kb}$ )

Additional file 7: NgBR (NUS1) mRNA expression data were retrieved from a gene-expression profiling dataset (225071_x from Kaplan-Meier Plot database) of 755 patients with ERa-positive breast cancer and 335 patients with ERa-positive breast cancer treated with endocrine therapy. Kaplan-Meier analysis revealed that the RFS in patients with ERa-positive breast cancer treated with endocrine therapy is significantly decreased in 167 patients with high NgBR expression in tumors as compared to 168 patients with low NgBR expression in tumors ( $p<0.05)$. (XLSX $32 \mathrm{~kb}$ )

Additional file 8: Survivin (BIRC5) mRNA expression data were retrieved from a gene-expression profiling dataset (202094_x from Kaplan-Meier Plot database) of 2046 patients with ERa-positive breast cancer and 928 patients with ERa-positive breast cancer treated with endocrine therapy. Kaplan-Meier analysis revealed significantly reduced relapse-free survival (RFS) $(p<0.05)$ in 1023 patients with ERa-positive breast cancer with high survivin expression in tumors as compared to 1023 patients with low survivin expression in tumors. (XLSX $70 \mathrm{~kb}$ )
Additional file 9: Survivin (BIRC5) mRNA expression data were retrieved from a gene-expression profiling dataset (202094_x from Kaplan-Meier Plot database) of 2046 patients with ERa-positive breast cancer and 928 patients with ERa-positive breast cancer treated with endocrine therapy. Kaplan-Meier analysis revealed that RFS in patients with ERa-positive breast cancer treated with endocrine therapy is significantly decreased in 463 patients with high survivin expression in tumors as compared to 465 patients with low NgBR expression in tumors $(p<0.05)$. (XLSX $60 \mathrm{~kb}$ )

Additional file 10: Figure S6. Relapse-free survival (RFS) in patients with ERa-positive breast cancer $(n=343)$. NgBR (NUS1) mRNA expression data were retrieved from the GSE6532 database. Kaplan-Meier analysis revealed significantly reduced RFS $(p<0.05)$ in patients with high NgBR expression in tumors $(n=189)$ as compared to patients with low $\mathrm{NgBR}$ expression in tumors $(n=154)$. (PDF $109 \mathrm{~kb})$

Additional file 11: NgBR (NUS1) mRNA expression data were retrieved from GSE6532 database. Kaplan-Meier analysis revealed significantly reduced relapse-free survival (RFS) $(p<0.05)$ in patients with high NgBR expression in tumors $(n=189)$ as compared to patients with low NgBR expression in tumors $(n=154)$. (XLS $67 \mathrm{~kb})$

\section{Abbreviations}

4-OHT: 4-Hydroxytamoxifen; Als: Aromatase inhibitors; AmNogo-B: Amino terminus of Nogo-B; DFS: Disease-free survival; DMEM: Dulbecco's modified Eagle's medium; EGF: Epidermal growth factor; EGFR: Epidermal growth factor receptor; EMT: Epithelial-mesenchymal transition; ERa: Estrogen receptor alpha; ERK: Extracellular signal-related kinase; FBS: Fetal bovine serum; GST: Glutathione; HA: Human influenza hemagglutinin; HER2: Human epidermal growth factor receptor 2; IHC: Immunohistochemistry; MAPK: Mitogen-activated protein kinase; MCF-7-TamR: Tamoxifen resistant MCF-7 cells; mERa: Membrane ERa; mRNA: Messenger RNA; NCCN: National Comprehensive Cancer Network; NgBR: Nogo-B receptor; NgBR-HA: Human influenza hemagglutinin (HA) tagged NgBR; NS: Non-silencing small interfering RNA; OS: Overall survival; PBS: Phosphate-buffered saline; PR: progesterone receptor; RBD: Ras-binding domain of Raf; RFS: Relapse-free survival; RTKs: Receptor tyrosine kinases; SERM: Selective estrogen receptor modulator; siNgBR: Nogo-B receptor small interfering RNA; siRNA: small interfering RNA; T47D-TamR: Tamoxifen-resistant T47D cells

\section{Acknowledgements}

We thank Meghann Sytsma at the Medical College of Wisconsin (MCW) for editing the manuscript and administrative support for this study.

\section{Funding}

This work is supported in part by start-up funds from the Division of Pediatric Surgery and Division of Pediatric Pathology, MCW and Advancing a Healthier Wisconsin endowment to MCW, NIH R01HL108938, NIH R01DK112971, Wisconsin Breast Cancer Showhouse (WBCS), Institutional Research Grant \# 86-004-26 from the American Cancer Society, Kathy Duffey Fogarty Award for breast cancer research, Rock River Research Foundation, State of Wisconsin Tax Check-off program for breast \& prostate cancer research, We Care Fund and Children's Hospital of Wisconsin Research Institute Pilot Innovative Research Grant to QRM; the National Natural Science Foundation of China (grant no. 81041098) and Bethune Program B of Jilin University (grant no. 2012217) to ZF; the National Natural Science Foundation of China (grant No. 81472734 and 81730071) to HZ.

\section{Availability of data and materials}

Microarray data presented in Fig. $7 \mathrm{~b}$ and $\mathrm{c}$ are available at the public database of Kaplan-Meier Plot (http://kmplot.com: NgBR/Nus1/225071_x; survivin/BIRC5/ 202094_x). Microarray data presented in Additional file 10: Figure S6 are available at the public database of NCBI's Gene Expression Omnibus (GEO) (https://www.ncbi.nim.nih.gov/geo, NgBR/Nus1: GSE6532). Please contact the corresponding authors for additional information and supporting data.

\section{Authors' contributions}

PG, XW, YJ, WH and YD conducted the experiments and data analysis; AS and YD collected tissue samples and performed histology analysis; PG and QRM designed the experiments and wrote the paper; ZF, HZ, AS, DS, MY, SL, $\mathrm{BH}$ and $\mathrm{GZ}$ provided reagents and edited the paper; QRM was responsible for overall integration and execution of the scientific approaches. All authors read and approved the final manuscript. 


\section{Ethics approval and consent to participate}

All patients signed a consent form allowing the study of their biological samples used in this study. The breast tissue specimens are anonymous. The study was approved by the ethical committee of the First Hospital of Jilin University (2017-346).

\section{Consent for publication}

Not applicable

\section{Competing interests}

The authors declare that they have no competing interests.

\section{Publisher's Note}

Springer Nature remains neutral with regard to jurisdictional claims in published maps and institutional affiliations.

\section{Author details}

'Department of Breast Surgery, The First Hospital of Jilin University, 71 Xinmin street, Changchun 130021, Jilin Province, China. ${ }^{2}$ Division of Pediatric Surgery, Department of Surgery, Children's Research Institute, Medical College of Wisconsin, 8701 W Watertown Plank Rd, Milwaukee, WI 53226, USA. ${ }^{3}$ Division of Pediatric Pathology, Department of Pathology, Children's Research Institute, Medical College of Wisconsin, 8701 W Watertown Plank Rd, Milwaukee, WI 53226, USA. " Department of Human Anatomy, Histology, and Embryology, Key Laboratory of Carcinogenesis and Translational Research (Ministry of Education) and State Key Laboratory of Natural and Biomimetic Drugs, Peking University Health Science Center, Beijing 100191, China. ${ }^{5}$ College of Life Sciences, Nankai University, 94 Weijin Road, Tianjin 300071, China

\section{Received: 15 December 2017 Accepted: 19 July 2018}

\section{Published online: 12 September 2018}

\section{References}

1. Jemal A, Bray F, Center MM, Ferlay J, Ward E, Forman D. Global cance statistics. CA Cancer J Clin. 2011;61 (2):69-90.

2. Siegel RL, Miller KD, Jemal A. Cancer statistics, 2017. CA Cancer J Clin. 2017:67(1):7-30

3. Harvey JM, Clark GM, Osborne CK, Allred DC. Estrogen receptor status by immunohistochemistry is superior to the ligand-binding assay for predicting response to adjuvant endocrine therapy in breast cancer. J Clin Oncol. 1999;17(5):1474-81.

4. Early Breast Cancer Trialists' Collaborative G. Effects of chemotherapy and hormonal therapy for early breast cancer on recurrence and 15-year survival: an overview of the randomised trials. Lancet. 2005;365(9472):1687-717.

5. Gradishar WJ, Anderson BO, Blair SL, Burstein HJ, Cyr A, Elias AD, Farrar WB, Forero A, Giordano SH, Goldstein LJ, et al. Breast cancer version 3.2014. J Natl Compr Cancer Netw. 2014:12(4):542-90.

6. Obrero M, Yu DV, Shapiro DJ. Estrogen receptor-dependent and estrogen receptor-independent pathways for tamoxifen and 4-hydroxytamoxifeninduced programmed cell death. J Biol Chem. 2002;277(47):45695-703.

7. Musgrove EA, Sutherland RL. Biological determinants of endocrine resistance in breast cancer. Nat Rev. 2009;9(9):631-43.

8. Arpino G, Green SJ, Allred DC, Lew D, Martino S, Osborne CK, Elledge RM. HER-2 amplification, HER-1 expression, and tamoxifen response in estrogen receptor-positive metastatic breast cancer: a southwest oncology group study. Clin Cancer Res. 2004;10(17):5670-6.

9. Zhen LL, Zhu X, Zheng W, Wang XY, Wu ZY. Involvement of epidermal growth factor receptor signaling pathway in tamoxifen resistance of MCF-7 cells. Ai Zheng. 2006;25(7):839-43.

10. Turner N, Pearson A, Sharpe R, Lambros M, Geyer F, Lopez-Garcia MA, Natrajan R, Marchio C, lorns E, Mackay A, et al. FGFR1 amplification drives endocrine therapy resistance and is a therapeutic target in breast cancer. Cancer Res. 2010;70(5):2085-94.

11. Huber AB, Weinmann O, Brosamle C, Oertle T, Schwab ME. Patterns of Nogo mRNA and protein expression in the developing and adult rat and after CNS lesions. J Neurosci. 2002;22(9):3553-67.

12. Miao RQ, Gao Y, Harrison KD, Prendergast J, Acevedo LM, Yu J, Hu F, Strittmatter SM, Sessa WC. Identification of a receptor necessary for Nogo-B stimulated chemotaxis and morphogenesis of endothelial cells. Proc Natl Acad Sci U S A. 2006;103(29):10997-1002.
13. Rana U, Liu Z, Kumar SN, Zhao B, Hu W, Bordas M, Cossette S, Szabo S, Foeckler J, Weiler $\mathrm{H}$, et al. Nogo-B receptor deficiency causes cerebral vasculature defects during embryonic development in mice. Dev Biol. 2016;410(2):190-201.

14. Park EJ, Grabinska KA, Guan Z, Sessa WC. NgBR is essential for endothelial cell glycosylation and vascular development. EMBO Rep. 2016;17(2):167-77.

15. Zhao B, Hu W, Kumar S, Gonyo P, Rana U, Liu Z, Wang B, Duong WQ, Yang $Z$, Williams $C L$, et al. The Nogo-B receptor promotes Ras plasma membrane localization and activation. Oncogene. 2017;36(24):3406-16.

16. Wang B, Zhao B, North P, Kong A, Huang J, Miao QR. Expression of NgBR is highly associated with estrogen receptor alpha and survivin in breast cancer. PLoS One. 2013;8(11):e78083.

17. Tamm I, Wang Y, Sausville E, Scudiero DA, Vigna N, Oltersdorf T, Reed JC. IAPfamily protein survivin inhibits caspase activity and apoptosis induced by Fas (CD95), Bax, caspases, and anticancer drugs. Cancer Res. 1998;58(23):5315-20.

18. Zhao B, Xu B, Hu W, Song C, Wang F, Liu Z, Ye M, Zou H, Miao QR. Comprehensive proteome quantification reveals $\mathrm{NgBR}$ as a new regulator for epithelial-mesenchymal transition of breast tumor cells. J Proteome. 2015;112:38-52

19. Wang L, Zhang X, Wang ZY. The Wilms' tumor suppressor WT1 regulates expression of members of the epidermal growth factor receptor (EGFR) and estrogen receptor in acquired tamoxifen resistance. Anticancer Res. 2010; 30(9):3637-42

20. Luo J, Wang W, Tang Y, Zhou D, Gao Y, Zhang Q, Zhou X, Zhu H, Xing L, Yu J. mRNA and methylation profiling of radioresistant esophageal cancer cells: the involvement of Sall2 in acquired aggressive phenotypes. J Cancer. 2017; 8(4):646-56.

21. Yuan J, Liu M, Yang L, Tu G, Zhu Q, Chen M, Cheng H, Luo H, Fu W, Li Z, et al. Acquisition of epithelial-mesenchymal transition phenotype in the tamoxifen-resistant breast cancer cell: a new role for $\mathrm{G}$ protein-coupled estrogen receptor in mediating tamoxifen resistance through cancerassociated fibroblast-derived fibronectin and beta1-integrin signaling pathway in tumor cells. Breast Cancer Res. 2015;17:69.

22. Thewes $V$, Simon R, Schroeter P, Schlotter M, Anzeneder T, Buttner R, Benes V, Sauter G, Burwinkel B, Nicholson Rl, et al. Reprogramming of the ERRalpha and ERalpha target gene landscape triggers tamoxifen resistance in breast cancer. Cancer Res. 2015;75(4):720-31.

23. Jin Y, Hu W, Liu T, Rana U, Aguilera-Barrantes I, Kong A, Kumar SN, Wang B, Gao $P$, Wang $X$, et al. Nogo-B receptor increases the resistance of estrogen receptor positive breast cancer to paclitaxel. Cancer Lett. 2018;419:233-44.

24. de Leeuw R, Neefjes J, Michalides R. A role for estrogen receptor phosphorylation in the resistance to tamoxifen. Int J Breast Cancer. 2011; 2011:232435.

25. Anbalagan M, Rowan BG. Estrogen receptor alpha phosphorylation and its functional impact in human breast cancer. Mol Cell Endocrinol. 2015 418(Pt 3):264-72.

26. Dai X, Cai C, Xiao F, Xiong Y, Huang Y, Zhang Q, Xiang Q, Lou G, Lian M, Su $Z$, et al. Identification of a novel aFGF-binding peptide with anti-tumor effect on breast cancer from phage display library. Biochem Biophys Res Commun. 2014:445(4):795-801.

27. Higuchi T, Endo M, Hanamura T, Gohno T, Niwa T, Yamaguchi Y, Horiguchi J, Hayashi S. Contribution of Estrone sulfate to cell proliferation in aromatase inhibitor (Al) -resistant, Hormone Receptor-Positive Breast Cancer. PloS One. 2016:11(5):e0155844

28. Paoletti C, Larios JM, Muniz MC, Aung K, Cannell EM, Darga EP, Kidwell KM, Thomas DG, Tokudome N, Brown ME, et al. Heterogeneous estrogen receptor expression in circulating tumor cells suggests diverse mechanisms of fulvestrant resistance. Mol Oncol. 2016;10(7):1078-85.

29. Kim J, Lee J, Jang SY, Kim C, Choi Y, Kim A. Anticancer effect of metformin on estrogen receptor-positive and tamoxifen-resistant breast cancer cell lines. Oncol Rep. 2016;35(5):2553-60.

30. Fan P, Agboke FA, Cunliffe HE, Ramos P, Jordan VC. A molecular model for the mechanism of acquired tamoxifen resistance in breast cancer. Eur J Cancer. 2014;50(16):2866-76.

31. Fan W, Chang J, Fu P. Endocrine therapy resistance in breast cancer: current status, possible mechanisms and overcoming strategies. Future Med Chem. 2015:7(12):1511-9.

32. Clarke R, Tyson JJ, Dixon JM. Endocrine resistance in breast cancer-an overview and update. Mol Cell Endocrinol. 2015:418(Pt 3):220-34

33. Schiff R, Massarweh SA, Shou J, Bharwani L, Mohsin SK, Osborne CK. Cross-talk between estrogen receptor and growth factor pathways as a molecular target for overcoming endocrine resistance. Clin Cancer Res. 2004;10(1 Pt 2):331S-6S. 
34. Xiong J, Su T, Qu Z, Yang Q, Wang Y, Li J, Zhou S. Triptolide has anticancer and chemosensitization effects by down-regulating Akt activation through the MDM2/REST pathway in human breast cancer. Oncotarget. 2016;7(17):23933-46.

35. Mayo LD, Donner DB. A phosphatidylinositol 3-kinase/Akt pathway promotes translocation of $\mathrm{Mdm} 2$ from the cytoplasm to the nucleus. Proc Natl Acad Sci U S A. 2001;98(20):11598-603.

36. Dong C, Zhao B, Long F, Liu Y, Liu Z, Li S, Yang X, Sun D, Wang H, Liu Q, et al. Nogo-B receptor promotes the chemoresistance of human hepatocellular carcinoma via the ubiquitination of p53 protein. Oncotarget. 2016;7(8):8850-65.

37. Muller PA, Vousden $\mathrm{KH}$. Mutant p53 in cancer: new functions and therapeutic opportunities. Cancer Cell. 2014;25(3):304-17.

38. Bailey ST, Shin H, Westerling T, Liu XS, Brown M. Estrogen receptor prevents p53-dependent apoptosis in breast cancer. Proc Natl Acad Sci U S A. 2012; 109(44):18060-5.

39. Mirza A, McGuirk M, Hockenberry TN, Wu Q, Ashar H, Black S, Wen SF, Wang $L$, Kirschmeier P, Bishop WR, et al. Human survivin is negatively regulated by wild-type p53 and participates in p53-dependent apoptotic pathway. Oncogene. 2002;21(17):2613-22.

40. Span PN, Tjan-Heijnen VC, Manders P, van Tienoven D, Lehr J, Sweep FC High survivin predicts a poor response to endocrine therapy, but a good response to chemotherapy in advanced breast cancer. Breast Cancer Res Treat. 2006;98(2):223-30.

41. Omerovic J, Laude AJ, Prior IA. Ras proteins: paradigms for compartmentalised and isoform-specific signalling. Cell Mol Life Sci. 2007; 64(19-20):2575-89.

42. Hancock JF. Ras proteins: different signals from different locations. Nat Rev Mol Cell Biol. 2003;4(5):373-84.

43. Buday L, Downward J. Many faces of Ras activation. Biochim Biophys Acta. 2008;1786(2):178-87.

44. McCubrey JA, Steelman LS, Abrams SL, Lee JT, Chang F, Bertrand FE, Navolanic PM, Terrian DM, Franklin RA, D'Assoro AB, et al. Roles of the RAF/ MEKJERK and PI3K/PTEN/AKT pathways in malignant transformation and drug resistance. Adv Enzym Regul. 2006;46:249-79.

45. McCubrey JA, Steelman LS, Chappell WH, Abrams SL, Wong EW, Chang F, Lehmann B, Terrian DM, Milella M, Tafuri A, et al. Roles of the Raf/MEK/ERK pathway in cell growth, malignant transformation and drug resistance. Biochim Biophys Acta. 2007;1773(8):1263-84

46. Karnoub AE, Weinberg RA. Ras oncogenes: split personalities. Nat Rev Mol Cell Biol. 2008;9(7):517-31.

47. Acconcia F, Kumar R. Signaling regulation of genomic and nongenomic functions of estrogen receptors. Cancer Lett. 2006;238(1):1-14.

48. Zhang $D$, Trudeau VL. Integration of membrane and nuclear estrogen receptor signaling. Comp Biochem Physiol A Mol Integr Physiol. 2006;144(3):306-15.

49. Soltysik K, Czekaj P. Membrane estrogen receptors - is it an alternative way of estrogen action? J Physiol Pharmacol. 2013;64(2):129-42.

50. Pritchard JE, Dillon PM, Conaway MR, Silva CM, Parsons SJ. A mechanistic study of the effect of doxorubicin/adriamycin on the estrogen response in a breast cancer model. Oncology. 2012;83(6):305-20.

51. Bunone G, Briand PA, Miksicek RJ, Picard D. Activation of the unliganded estrogen receptor by EGF involves the MAP kinase pathway and direct phosphorylation. EMBO J. 1996;15(9):2174-83.

52. Guo S, Li Y, Tong Q, Gu F, Zhu T, Fu L, Yang S. deltaEF1 down-regulates ER-alpha expression and confers tamoxifen resistance in breast cancer. PLoS One. 2012;7(12):e52380

53. Knowlden JM, Hutcheson IR, Jones HE, Madden T, Gee JM, Harper ME, Barrow D, Wakeling AE, Nicholson Rl. Elevated levels of epidermal growth factor receptor/c-erbB2 heterodimers mediate an autocrine growth regulatory pathway in tamoxifen-resistant MCF-7 cells. Endocrinology. 2003;144(3):1032-44.

54. Oh AS, Lorant LA, Holloway JN, Miller DL, Kern FG, El-Ashry D. Hyperactivation of MAPK induces loss of ERalpha expression in breast cancer cells. Mol Endocrinol. 2001;15(8):1344-59.

55. Bayliss J, Hilger A, Vishnu P, Diehl K, El-Ashry D. Reversal of the estrogen receptor negative phenotype in breast cancer and restoration of antiestrogen response. Clin Cancer Res. 2007;13(23):7029-36.

\section{Ready to submit your research? Choose BMC and benefit from:}

- fast, convenient online submission

- thorough peer review by experienced researchers in your field

- rapid publication on acceptance

- support for research data, including large and complex data types

- gold Open Access which fosters wider collaboration and increased citations

- maximum visibility for your research: over $100 \mathrm{M}$ website views per year

At BMC, research is always in progress.

Learn more biomedcentral.com/submissions 